\title{
Quantum Statistical Theory of Superconductivity in $\mathrm{MgB}_{2}$
}

\author{
S. Fujita1, A. Suzuki'2, Y. Takato ${ }^{3}$ \\ ${ }^{1}$ Department of Physics, University at Buffalo, State University of New York, Buffalo, NY, USA \\ ${ }^{2}$ Department of Physics, Faculty of Science, Tokyo University of Science, Tokyo, Japan \\ ${ }^{3}$ Okinawa Institute of Science and Technology Graduate University, Okinawa, Japan \\ Email:fujita@buffalo.edu,asuzuki@rs.kagu.tus.ac.jp,yoichi.takato@oist.jp
}

Received 30 June 2016; accepted 28 August 2016; published 31 August 2016

Copyright (C) 2016 by authors and Scientific Research Publishing Inc.

This work is licensed under the Creative Commons Attribution International License (CC BY).

http://creativecommons.org/licenses/by/4.0/

(c) (i) Open Access

\section{Abstract}

A quantum statistical theory of the superconductivity in $\mathrm{MgB}_{2}$ is developed regarding it as a member of the graphite intercalation compound. The superconducting temperature $T_{\mathrm{c}}$ for $\mathbf{M g B}_{2}$, $\mathrm{C}_{8} \mathrm{~K} \equiv \mathrm{KC}_{8}, \mathrm{CaC}_{6}$, are $39 \mathrm{~K}, 0.6 \mathrm{~K}, 11.5 \mathrm{~K}$, respectively. The differences arise from the lattice structures. In the plane perpendicular to the c-axis, B's form a honeycomb lattice with the nearest neighbour distance $a_{0}$ while Mg's form a base-hexagonal lattice with the nearest neighbour distance $\sqrt{3} a_{0}$ above and below the B-plane distanced by $c_{0}$. The more compact B-plane becomes superconducting due to the electron-phonon attraction. Starting with the generalized BardeenCooper-Schrieffer (BCS) Hamiltonian and solving the generalized Cooper equation, we obtain a linear dispersion relation $\varepsilon=c p$ for moving Cooper pairs. The superconducting temperature $T_{\mathrm{c}}$ identified as the Bose-Einstein condensation temperature of the Cooper pairs in two dimensions is given by $T_{\mathrm{c}}=1.954 \hbar c n_{0}^{1 / 2} k_{\mathrm{B}}^{-1}$, where $n_{0}$ is the Cooper pair density, $k_{\mathrm{B}}$ the Boltzmann constant. The lattices of $\mathrm{KC}_{8}$ and $\mathrm{CaC}_{6}$ are clearly specified.

\section{Keywords}

Crystal Structure, BCS Hamiltonian, Electron-Phonon Interaction, Cooper Pairs, Bose-Einstein Condensation, Superconductivity

\section{Introduction}

Nagamatsu et al. [1] reported in 2001 superconductivity at $39 \mathrm{~K}$ in magnesium diboride $\mathrm{MgB}_{2} . \mathrm{MgB}_{2}$ forms a lattice closely related to that of a graphite intercalation compound (GIC). It is similar to $\mathrm{NaC}_{2}$ composition- wise, but the 
lattice structures are distinct as shown below. The superconducting temperatures $T_{\mathrm{c}}$ of $\mathrm{MgB}_{2}$ and $\mathrm{NaC}_{2}$ are $39 \mathrm{~K}$ and $5 \mathrm{~K}$, respectively. This difference arises from the lattice structures. Canfield and Crabtree [2] wrote a comprehensive review in Physics Today (2003). From the isotope effect study [2] [3] they concluded that the B-plane contains a honeycomb lattice which becomes superconducting at $0 \mathrm{~K}$, while the Mg-plane is base-hexagonal and is metallic. Their conjectured lattice structure of $\mathrm{MgB}_{2}$ is shown in Ref. 2, Figure 3. The superconducting state occurs at $0 \mathrm{~K}$, where the entropy of an electron-phonon system vanishes. The third law of the thermodynamics applies. The crystal must be specified with the location of all atoms. Ref. 2, Figure 3 contains only the B-lattice and Mg-lattice with unspecifyed lattice constants. We must specify lattice systems with basic lattice units with the lattice constants. A currently presumed lattice for $\mathrm{MgB}_{2}$ [4] is a fully intercalated graphite compound similar to that in $\mathrm{NaC}_{2}$. We propose a different lattice. The two lattices have the same first neighbour configurations but different second nearest neighbours. Our proposed lattice has a lower Coulomb energy and should be realized in practice.

We shall develop a quantum statistical theory of the superconductivity in $\mathrm{MgB}_{2}$, starting with a generalized Bardeen-Cooper-Schrieffer (BCS) Hamiltonian [5] and calculateing everything using the standard quantum statistical methods. Canfield-Crabtree's and our lattices have nearly the same energies if the first neighbour configurations are examined. The second neighbour confi- gurations are different. Each $\mathrm{B}^{+}$in our lattice is surrounded by six $\mathrm{Mg}^{+}$'s while each $\mathrm{B}^{+}$in Canfield-Crabtree's lattice is surrounded by three $\mathrm{Mg}^{+}$. Hence our lattice is more stable. In the course of the development, we clearly specify the lattices of $\mathrm{C}_{8} \mathrm{~K} \equiv \mathrm{KC}_{8}$ and $\mathrm{CaC}_{6}$.

\section{Electron Dynamics}

Following Ashcroft and Mermin (AM) [6], we assume that "electrons" and "holes" in solids run as wave packets (not point-particles). We adopt the semiclassical model of electron dynamics in solids [6]. It is necessary to introduce a $k$-vector:

$$
\boldsymbol{k}=k_{x} \hat{\boldsymbol{e}}_{x}+k_{y} \hat{\boldsymbol{e}}_{y}+k_{z} \hat{\boldsymbol{e}}_{z},
$$

where $\hat{\boldsymbol{e}}_{x}, \hat{\boldsymbol{e}}_{y}, \hat{\boldsymbol{e}}_{z}$ are the orthonormal vectors, since the $k$-vectors are involved in the semiclassical equation of motion:

$$
\hbar \dot{\boldsymbol{k}} \equiv \hbar \frac{\mathrm{d} \boldsymbol{k}}{\mathrm{d} t}=q(\boldsymbol{E}+\boldsymbol{v} \times \boldsymbol{B}),
$$

where $q=-e$ is the electron charge, and $\boldsymbol{E}$ and $\boldsymbol{B}$ are the electric and magnetic fields, respectively. The vector

$$
\boldsymbol{v} \equiv \frac{1}{\hbar} \frac{\partial \varepsilon}{\partial \boldsymbol{k}}
$$

is the electron velocity, where $\varepsilon=\varepsilon(\boldsymbol{k})$ is the energy.

If the electron is in a continuous energy range (energy band), then it will be accelerated by the electric force $q \boldsymbol{E}$, and the material is a conductor. If the electron's energy is in a forbidden band (energy gap), it does not move under a small electric force, and the material is insulator. If the acceleration occurs only for a mean free time (inverse of scattering frequency) $\tau$, the conductivity $\sigma$ for a simple metal is given by Drude's formula [6]:

$$
\sigma=q^{2} n \tau / m^{*}
$$

where $n$ is the electron density and $m^{*}$ the effective mass.

We consider a graphene which forms a 2D honeycomb lattice. The Wigner-Seitz (WS) unit cell, a rhombus, contains two C's. We showed in our earlier work [7] [8] that graphene has "electrons" and "holes" based on the rectangular unit cell. We briefly review our calculations below. We assume that the "electron" ("hole") wave packet has the charge $-e(+e)$ and a size of the rectangular unit cell, generated above (below) the Fermi energy $\varepsilon_{\mathrm{F}}$. We showed [7] earlier that a) the "electron" and "hole" have different charge distributions and different effective masses; b) that the "electrons" and "holes" move in different easy channels; c) that the "electrons" and "holes" are thermally excited with different activation energies $\left(\varepsilon_{1}, \varepsilon_{2}\right)$, and d) that the "electron" activation energy $\varepsilon_{1}$ is smaller than the "hole" activation energy $\varepsilon_{2}$ :

$$
\varepsilon_{1}<\varepsilon_{2} \text {. }
$$

Hence, "electrons" are the majority carriers in graphene. The thermally activated electron densities are given by 


$$
n_{j}(T)=n_{j} \mathrm{e}^{-\varepsilon_{j} / k_{\mathrm{B}} T}, \quad n_{j}=\text { constant },
$$

where $j=1$ and 2 represent the "electron" and "hole", respectively.

\section{Lattice Structures in $\mathrm{C}_{8} \mathrm{~K}=\mathrm{KC}_{8}, \mathrm{CaC}_{6}$ and $\mathrm{MgB}_{2}$}

Graphite is composed of graphene layers stacked in the manner $\mathrm{ABAB} \cdots$ along the $c$-axis. We may choose an orthogonal unit cell shown in Figure 1.

The carbons (circles) in the A (B) planes are shown in dark (light) gray circles.

The unit cell contains $16 \mathrm{C}$ 's. The two rectangles (white solid lines) are stacked vertically with the interlayer separation, $c_{0}=3.35 \AA$, much greater than the nearest neighbour distance between two C's, $a_{0}=1.42 \AA$ :

$$
c_{0} \gg a_{0} \text {. }
$$

The unit cell has three side-lengths:

$$
b_{1}=3 a_{0}, \quad b_{2}=2 \sqrt{3} a_{0}, \quad b_{3}=2 c_{0} .
$$

Clearly, the system is periodic along the orthogonal directions with the three periods $\left(b_{1}, b_{2}, b_{3}\right)$ given in Equation (8). Hence, the system can be considered as orthorhombic with the sides $\left(b_{1}, b_{2}, b_{3}\right), b_{1} \neq b_{2}, b_{1} \neq b_{3}$, $b_{2} \neq b_{3}$.

The negatively charged "electron" (with the charge $-e$ ) in graphite are welcomed by the positively charged $\mathrm{C}^{+}$when moving vertically up or down in the plane. Then, the easy direction for the "electrons" is vertical. The easy direction for the "holes" is horizontal. There are no hindering hills for "holes" moving horizontally. Hence, the "electron" in graphite has the lower activation energy $\varepsilon$ than the "hole": $\varepsilon_{1}<\varepsilon_{2}$. Then, "electrons" are the majority carriers in graphite. The thermoelectric power (Seebeck coefficient) measurements by Kang et al. [9] show that the majority carriers in graphite are "electrons" in agreement with our theory.

We now consider GIC. Let us first take $\mathrm{C}_{8} \mathrm{~K}$. The $\mathrm{K}^{+}$ions should enter as interstitials and occupy the sites away from the positive ions $\mathrm{C}^{+}$. We see in Figure 1 that the center of the unit cell is empty. Each $\mathrm{K}^{+}$should occupy the midpoint between two graphene layers. The 3D unit cell contains $16 \mathrm{C}$ 's and $2 \mathrm{~K}$ 's. Alkali metal $\mathrm{GIC}$, including $\mathrm{C}_{8} \mathrm{Li}, \mathrm{C}_{8} \mathrm{Rb}$, should form similar lattices. Next we condier $\mathrm{C}_{6} \mathrm{Ca}$. Carbons $(\mathrm{C})$ in graphite form a honeycomb lattice in the A plane as shown in Figure 1. There are eight (8) C's and four (4) hexagon centers, (two full circles, two half-circles and four quarter-circles). If we fill the hexagon centers with C's, then we obtain twelve (12) C's in the 2D unit cell. Similarly the configuration of the B-plane and that one below is prescribed. There are $2 \times 12 \mathrm{C}$ 's in the planes $\mathrm{A}$ and $\mathrm{B}$, and $2 \times 2 \mathrm{Ca}$ 's between the planes for $\mathrm{C}_{6} \mathrm{Ca}$. The composition ratio 6:1 is correct. After the $\mathrm{C}$-filling, the $\mathrm{C}$-plane becomes primitive (base)-hexagonal and has a $60^{\circ}$ rotation symmetry. The primitive unit cell contains six (6) C's. Two Ca's are likely to occupy below the centers of the primitive cells located at the two-light gray circles in Figure 1. A real 3D $\mathrm{C}_{6} \mathrm{Ca}$ is obtained by stacking the $\mathrm{C}_{6} \mathrm{Ca}$ sheets in the manner $\mathrm{ABAB} \ldots$ We note that the structure of $\mathrm{C}_{6} \mathrm{Ca}$ is significantly more compact than that of $\mathrm{C}_{8} \mathrm{~K} . \mathrm{C}_{6} \mathrm{Yb}$ should have a similar lattice structure. $\mathrm{GIC}_{4} \mathrm{Na}\left(\mathrm{C}_{3} \mathrm{~K}, \mathrm{C}_{2} \mathrm{Na}\right)$ should have the same $12 \mathrm{C}$-sheets and $3 \mathrm{Na}(4 \mathrm{~K}, 6 \mathrm{Na})$ intersheets.

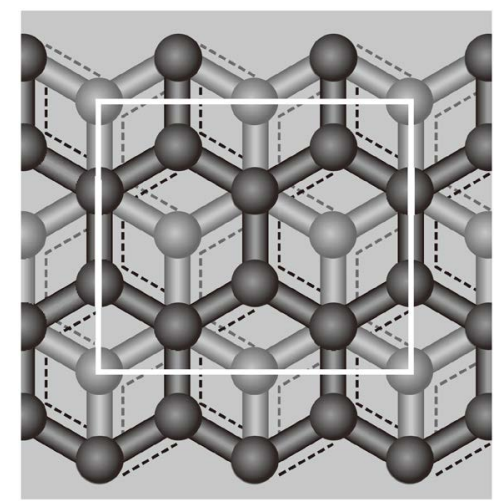

Figure 1. An orthogonal unit cell (white solid lines) viewed from the top for graphite. The carbons (circles) in the A (B) planes are shown in dark (light) gray circles. 
Consider now $\mathrm{MgB}_{2}$. It is only natural to start with the B-plane since this plane becomes superconducting at 0 K. Let us look at the top sheet in Figure 1. Within the white rectangle, there are eight (8) balls and four (4) vacant hexagons. If B's occupy the ball sites and Mg's occupy the hexagon-centers sites in the neighbour sheet above (or below), then we obtain the most likely lattice. The composition ratio 2:1 is correct. The B-plane contains a honeycomb lattice with a nearest neighbour distance $a_{0}$ with a $120^{\circ}$ rotation symmetry. The Mg-plane contains a base-hexagonal lattice with the nearest neighbour distance $\sqrt{3} a_{0}$. Crystals Mg and B are known divalent and trivalent hexagonal metals [6]. Since the B-plane in $\mathrm{MgB}_{2}$ is more compact with the smaller lattice constant, the B-plane is likely to become superconducting at the lowest temperatures. Note that all ions position are specified. Ions $\mathrm{Mg}^{+}$and $\mathrm{B}^{+}$are positively charged so that they tend to stay away among and between them.

Our lattice and Canfield-Crabtree's are different in the second nearest neighbour configuration. Each $\mathrm{B}^{+}$in our lattice is surrounded by six $\mathrm{Mg}^{+}$while each $\mathrm{B}^{+}$in Canfield-Crabtree's lattice is surrounded by three $\mathrm{Mg}^{+}$. Hence our lattice is more stable. The B-plane contains a honeycomb lattice of B's for both. Our Mg-plane contains a base-hexagonal lattice of the nearest neighbour distance $\sqrt{3} a_{0}$. In summary we found that a) the B-honeycomb lattice has smaller nearest neighbour distance $a_{0}$ than the Mg hexagonal lattice with the nearest neighbour distance $\sqrt{3} a_{0}$. The more compact means the higher conduction electron density; b) The centers of mass $(\mathrm{CM})$ of hexagons are displaced upward by a short distance $a_{0} / 2$ to take advantage of the smaller repulsive Coulomb energy. There is a mismatch between the B-plane and the Mg-plane centers, a unique feature for $\mathrm{MgB}_{2}$.

\section{The Hamiltonian}

The countability and statistics of the fluxons (magnetic flux quanta) are the fundamental particle properties. We postulate that the fluxon is a half-spin fermion with zero mass and zero charge.

We assume that the magnetic field $\boldsymbol{B}$ is applied perpendicular to the graphene plane. The 2D Landau level energy,

$$
\varepsilon=\hbar \omega_{\mathrm{c}}\left(N_{\mathrm{L}}+\frac{1}{2}\right), \quad \omega_{\mathrm{c}} \equiv e B / m^{*},
$$

with the states $\left(N_{\mathrm{L}}, k_{y}\right), N_{\mathrm{L}}=0,1,2, \cdots$, have a great degeneracy (no $k_{y}$-dependence). The $m^{*}$ is the effective mass of an "electron". Following Zhang, Hansson and Kivelson [10], we introduce composite (c-) particles. The Center-of-Mass (CM) of any c-particle moves as a fermion or a boson. That is, the eigenvalues of the $\mathrm{CM}$ momentum are limited to 0 or 1 (unlimited) if the composite contains an odd (even) number of elementary fermions. This rule is known as the Ehrenfest-Oppenheimer-Bethe's (EOB's) rule [11]. Hence the $\mathrm{CM}$ motion of the composite containing an electron and $Q$ fluxons is bosonic (fermionic) if $Q$ is odd (even). The system of the c-bosons condenses below some critical temperature $T_{\mathrm{c}}$ and exhibits a superconducting state while the system of c-fermions shows a Fermi liquid behavior.

A longitudinal phonon, acoustic or optical, generates a charge density wave, which affects the electron (fluxon) motion through the charge displacement (current). Let us first consider the case of superconductivity. The phonon exchange between two electrons shown in Figure 2 generates a transition in the electron states with the effective interaction

$$
\mathcal{V}_{\text {eff }}=\left|V_{q}\right|^{2} \frac{\hbar \omega_{q}}{\left(\varepsilon_{|k+q|}-\varepsilon_{k}\right)^{2}-\left(\hbar \omega_{q}\right)^{2}},
$$

where $\varepsilon_{k}$ is the electron energy, $\hbar \omega_{q}$ the phonon energy, and $V_{q}$ the electron-phonon interaction strength.

An electric current $(\boldsymbol{J})$ loop generates a magnetic field (flux) ${ }^{q} \boldsymbol{B}$ while a magnetic flux $\boldsymbol{B}$ is surrounded by diamagnetic currents $\boldsymbol{J}$. Thus the currents $\boldsymbol{J}$ and the magnetic field $\boldsymbol{B}$ are coupled. The exchange of a phonon between an electron and a fluxon also generates a transition in the electron states with the effective interaction:

$$
\left|V_{q} V_{q}^{\prime}\right| \frac{\hbar \omega_{q}}{\left(\varepsilon_{|k+q|}-\varepsilon_{k}\right)^{2}-\left(\hbar \omega_{q}\right)^{2}},
$$




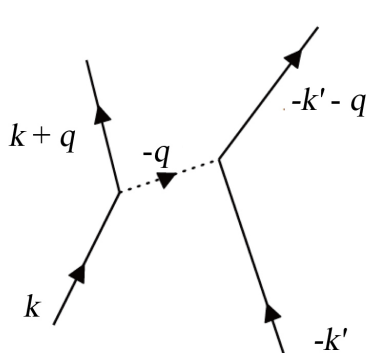

(a)

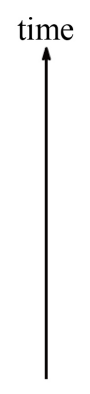

(b)

\begin{abstract}
Figure 2. Electron's (phonon's) motions are represented by solid (dotted) lines, and the time is measured upwards. Two phonon exchange processes (a) and (b) generate the momenta change from the initial pair state $\left(\boldsymbol{k},-\boldsymbol{k}^{\prime}\right)$ to the final states $\left(\boldsymbol{k}+\boldsymbol{q},-\boldsymbol{k}^{\prime}-\boldsymbol{q}\right)$.
\end{abstract}

where $V_{q}^{\prime}\left(V_{q}\right)$ is the fluxon-phonon (electron-phonon) interaction constant. The Landau oscillator quantum number $N_{\mathrm{L}}$ is omitted; the bold $\boldsymbol{k}$ denotes the momentum $\left(k_{y}\right)$ and the italic $k(=|\boldsymbol{k}|)$ the magnitude. There are two processes, one with the absorption of a phonon with momentum $\boldsymbol{q}$ and the other with the emission of a phonon with momentum $-q$, see Figure 3(a) and Figure 3(b), which contribute to the effective interaction with the energy denominators $\left(\varepsilon_{|k+q|}-\varepsilon_{k}-\hbar \omega_{q}\right)^{-1}$ and $\left(\varepsilon_{|k+q|}-\varepsilon_{k}+\hbar \omega_{q}\right)^{-1}$, generating Equation (11). The interaction is attractive (negative) and most effective when the states before and after the exchange have the same energy $\left(\varepsilon_{|k+q|}-\varepsilon_{k}=0\right)$ as for the degenerate 2D LL.

BCS [5] assumed the existence of Cooper pairs [12] in a superconductor, and wrote down a Hamiltonian containing the "electron" and "hole" kinetic energies and the pairing interaction Hamiltonian with the phonon variables eliminated. We start with a BCS-like Hamiltonian $\mathcal{H}$ for the QHE: [13]

$$
\begin{aligned}
\mathcal{H}= & \sum_{k}^{\prime} \sum_{s} \varepsilon_{k}^{(1)} n_{k s}^{(1)}+\sum_{k}^{\prime} \sum_{s} \varepsilon_{k}^{(2)} n_{k s}^{(2)}+\sum_{k}^{\prime} \sum_{s} \varepsilon_{k}^{(3)} n_{k s}^{(3)} \\
& -\sum_{q}^{\prime} \sum_{k}^{\prime} \sum_{\boldsymbol{k}^{\prime}}^{\prime} \sum_{s} v_{0}\left[B_{\boldsymbol{k}^{\prime} \boldsymbol{q} s}^{(1) \dagger} B_{\boldsymbol{k} \boldsymbol{q} s}^{(1)}+B_{\boldsymbol{k}^{\prime} \boldsymbol{q} \boldsymbol{s}}^{(1) \dagger} B_{\boldsymbol{k} \boldsymbol{q} s}^{(2) \dagger}+B_{\boldsymbol{k}^{\prime} \boldsymbol{q} s}^{(2)} B_{k \boldsymbol{k} s}^{(1)}+B_{\boldsymbol{k}^{\prime} \boldsymbol{q} s}^{(2)} B_{\boldsymbol{k} \boldsymbol{q} s}^{(2) \dagger}\right],
\end{aligned}
$$

where $n_{k s}^{(j)} \equiv c_{k s}^{(j) \dagger} c_{k s}^{(j)}$ is the number operator for the "electron" (1) ("hole" (2), fluxon (3)) at momentum $\boldsymbol{k}$ and spin $s$ with the energy $\varepsilon_{k s}^{(j)}$, with annihilation (creation) operators $c\left(c^{\dagger}\right)$ satisfying the Fermi anticommutation rules:

$$
\left\{c_{k s}^{(i)}, c_{\boldsymbol{k}^{\prime} s^{\prime}}^{(j)}\right\} \equiv c_{\boldsymbol{k} s}^{(i)} c_{\boldsymbol{k}^{\prime} s^{\prime}}^{(j) \dagger}+c_{\boldsymbol{k}^{\prime} s^{\prime}}^{(j) \dagger} c_{\boldsymbol{k} s}^{(i)}=\delta_{\boldsymbol{k}, \boldsymbol{k}^{\prime}} \delta_{s, s^{\prime}} \delta_{i, j}, \quad\left\{c_{k s}^{(i)}, c_{\boldsymbol{k}^{\prime} s^{\prime}}^{(j)}\right\}=0 .
$$

The fluxon number operator $n_{k s}^{(3)}$ is represented by $a_{k s}^{\dagger} a_{k s}$ with $a\left(a^{\dagger}\right)$ satisfying the anti-commutation rules:

$$
\left\{a_{k s}, a_{k^{\prime} s^{\prime}}^{\dagger}\right\}=\delta_{k, k^{\prime}} \delta_{s, s^{\prime}}, \quad\left\{a_{k s}, a_{k^{\prime} s^{\prime}}\right\}=0 .
$$

The phonon exchange can create electron-fluxon composites, bosonic or fermionic, depending on the number of fluxons. The CM of any composite moves as a fermion (boson) if it contains an odd (even) numbers of elementary fermions. The electron (hole)-type c-particles carry negative (positive) charge. Electron (hole)-type Cooper-pair-like c-bosons are generated by the phonon-exchange attraction from a pair of electron (hole)-type c-fermions. The pair operators $B$ are defined by

$$
B_{\boldsymbol{k} \boldsymbol{q}, s}^{(1) \dagger} \equiv c_{\boldsymbol{k}+\boldsymbol{q} / 2,-s}^{(1) \dagger} a_{-\boldsymbol{k}+\boldsymbol{q} / 2,-s}^{\dagger}, \quad B_{\boldsymbol{k} \boldsymbol{q}, s}^{(2)} \equiv a_{-\boldsymbol{k}+\boldsymbol{q} / 2,-s} c_{\boldsymbol{k}+\boldsymbol{q} / 2,-s}^{(2)} .
$$

The prime on the summation in Equation (12) means the restriction:

$$
0<\varepsilon_{k s}^{(j)}<\hbar \omega_{D}, \quad \omega_{D}=\text { Debye frequency. }
$$




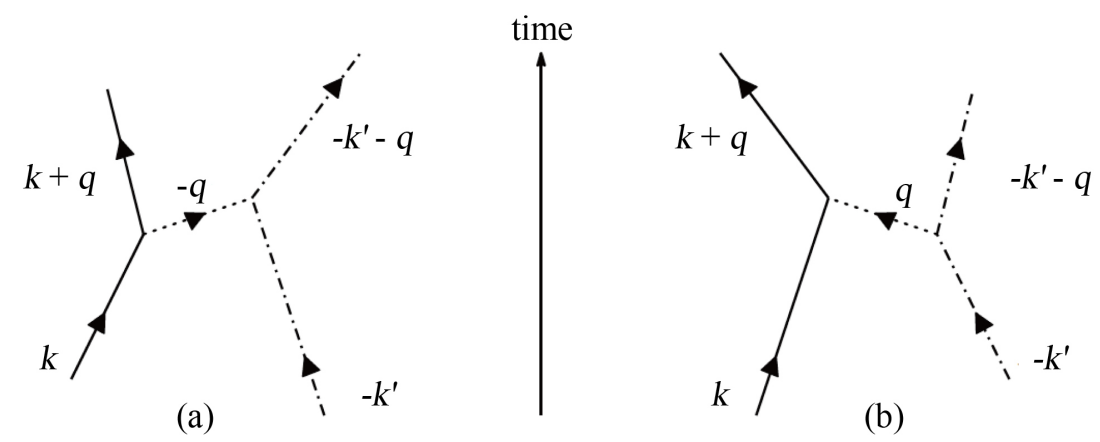

Figure 3. Fluxon's motion is represented by dot-dashed lines. The phonon exchange processes (a) and (b) generate the momenta change from the initial (electron, fluxon) state $\left(\boldsymbol{k},-\boldsymbol{k}^{\prime}\right)$ to the final states $\left(\boldsymbol{k}+\boldsymbol{q},-\boldsymbol{k}^{\prime}-\boldsymbol{q}\right)$.

The pairing interaction terms in Equation (12) conserve the charge. The term $-v_{0} B_{k^{\prime} q}^{(1) \dagger} B_{k q s}^{(1)}$, where $v_{0}$ is the pairing strength, generates a transition in electron-type c-particle states. Similarly, the exchange of a phonon generates a transition between hole-type c-particle states, represented by $-v_{0} B_{k^{\prime} s_{s}}^{(2) \dagger} B_{k q s}^{(2) \dagger}$. The phonon exchange can also pair-create (pair-annihilate) electron (hole)-type c-boson pairs, and the effects of these processes are represented by $-v_{0} B_{\boldsymbol{k}^{\prime} \boldsymbol{s}}^{(1) \dagger} B_{\boldsymbol{k} \boldsymbol{s} s}^{(2) \dagger}\left(-v_{0} B_{\boldsymbol{k} \boldsymbol{s} s}^{(1)} B_{\boldsymbol{k} \boldsymbol{s} s}^{(2)}\right)$.

The Cooper pair, also called the pairon, is formed from two "electrons" (or "holes"). The pairons move as bosons, which are shown in Appendix. Likewise the c-bosons may be formed by the phonon-exchange attraction from two like-charge c-fermions. If the density of the c-bosons is high enough, then the c-bosons will be Bosecondensed and exhibit a superconductivity.

The pairing interaction terms in Equation (12) are formally identical with those in the generalized BCS Hamiltonian [13]. Only we deal here with c-fermions instead of conduction electrons.

The c-bosons, having the linear dispersion relation, can move in all directions in the plane with the constant speed $(2 / \pi) v_{\mathrm{F}}^{(j)}$ [13]. For completeness we show the linear dispersion relation in Appendix. The supercurrent is generated by $\mp$ c-bosons monochromatically condensed, running along the sample length. The supercurrent density (magnitude) $j$, calculated by the rule:

$$
j=\left(e^{*}: \text { carrier charge }\right) \times\left(n_{0}: \text { carrier density }\right) \times\left(v_{\mathrm{d}}: \text { carrier drift velocity }\right)
$$

is given by

$$
j \equiv e^{*} n_{0} v_{\mathrm{d}}=e^{*} n_{0} \frac{2}{\pi}\left|v_{\mathrm{F}}^{(1)}-v_{\mathrm{F}}^{(2)}\right|,
$$

where $e^{*}$ is the effective charge of carriers. The induced Hall field (magnitude) $E_{\mathrm{H}}$ equals $v_{\mathrm{d}} B$. The magnetic flux is quantized:

$$
B=n_{\phi} \Phi_{0}, \quad n_{\phi}=N_{\phi} / A
$$

where $N_{\phi}$ is the fluxon number, and

$$
\Phi_{0} \equiv h / e
$$

Hence we obtain the Hall resistivity as

$$
\rho_{\mathrm{H}} \equiv \frac{E_{\mathrm{H}}}{j}=\frac{v_{\mathrm{d}} B}{e^{*} n_{0} v_{\mathrm{d}}}=\frac{1}{e^{*} n_{0}} n_{\phi} \Phi_{0}=\frac{n_{\phi}}{e^{*} n_{0}}\left(\frac{h}{e}\right) .
$$

For the integer QHE at $v=1$, we have $e^{*}=e, n_{\phi}=n_{0}$. Hence, we obtain $\rho_{\mathrm{H}}=h / e^{2}$, the plateau value observed.

The supercurrent generated by equal numbers of \pm c-bosons condensed monochromatically is neutral. This is reflected in our calculations in Equation (18). The supercondensate whose motion generates a supercurrent must 
be neutral. If it has a charge, it would then be accelerated indefinitely by any external electric field because the impurities and phonons cannot stop the supercurrent to grow. That is, the circuit containing a superconducting sample and a battery must be burnt out if the supercondensate is not neutral. In the calculation of $\rho_{\mathrm{H}}$ in Equation (21), we used the unaveraged drift velocity $v_{\mathrm{d}}=(2 / \pi)\left|v_{\mathrm{F}}^{(1)}-v_{\mathrm{F}}^{(2)}\right|$, which is significant. Only the unaveraged drift velocity cancels out $v_{\mathrm{d}}$ exactly from numerator/denominator, leading to an exceedingly accurate plateau value.

We now extend our theory to include elementary fermions (electron, fluxon) as members of the c-fermion set. We can then treat the 2D superconductivity and the QHE in a unified manner. The c-boson containing one electron and one fluxon can be used to describe the principal QHE. Important pairings and effects are listed below: a) a pair of conduction electrons, superconductivity; b) c-fermions and fluxon, QHE; c) a pair of likecharge conduction electrons with two fluxons, QHE in graphene.

\section{Superconductivity in $\mathrm{C}_{8} \mathrm{~K}, \mathrm{CaC}_{6}$ and $\mathrm{MgB}_{2}$}

\subsection{Preliminaries}

\subsection{1. "Electrons", "Holes" and "Phonons"}

The conduction electrons ("electrons", "holes") are excited based on the orthogonal unit cells. As mentioned earlier the "electrons" are the majority carriers in both graphene and graphite. The excitation energy for the "electrons" is smaller than for the "holes". Phonons are generated based on the same orthogonal unit cells. Phonons are bosons, and hence can be generated with no activation energies. The phonons are distributed, following the Planck distribution function:

$$
f_{\mathrm{P}}(\varepsilon)=\left[\exp \left(\varepsilon / k_{\mathrm{B}} T\right)-1\right]^{-1},
$$

which is a sole function of the Kelvin temperature $T$.

As an example consider acoustic phonons with a linear dispersion relation:

$$
\varepsilon=s p,
$$

where $s$ is the sound speed. The phonon size may be characterized by the average wave length:

$$
\lambda=s / k \text {. }
$$

The average size of phonons at the room temperature is greater by a few orders of magnitudes than the electron size.

\subsubsection{The Ground-State Cooper Pair (Pairon) Energy}

Cooper solved the Cooper equation [Ref. 12, Equation (1)] with a negative interaction energy constant, $-v_{0}$, and obtained the ground-state pairon energy:

$$
w_{0}=\frac{-2 \hbar \omega_{\mathrm{D}}}{\exp \left[2 / v_{0} \mathcal{D}(0)\right]-1}
$$

where $\omega_{\mathrm{D}}=$ Deby frequency, $\mathcal{D}(0)=\mathcal{D}\left(\varepsilon_{\mathrm{F}}=0\right)=$ density of states at the Fermi energy. The energy $w_{0}$ is singular at $v_{0}=0$. Hence, this bound-state energy $w_{0}$ cannot be obtained by a perturbation theory. For illustration, consider a hydrogen atom levels problem with a negative Coulomb interaction $\mathcal{V}$. The bound states and energies are obtained by directly solving the Schrödinger equation with the full Hamiltonian containing the kinetic energy and the interaction energy $\mathcal{V}$. The Cooper pair is formed from two "electrons" (or "holes"). Likewise the c-bosons may be formed by the phonon-exchange attraction from c-fermions and fluxons. If the density of the c-bosons is high enough, then the c-bosons will be Bose-condensed and exhibit a superconductivity.

\subsection{The Superconductivity in GIC and $\mathrm{MgB}_{2}$}

The superconductivity occurs only in regular crystals. That is, it occurs only in crystals and not in liquids. $\mathrm{C}_{8} \mathrm{~K}$ and graphene have a $120^{\circ}$ rotation symmetry. $\mathrm{C}_{6} \mathrm{Ca}$ has a base-hexagonal $\left(60^{\circ}\right.$ rotation) symmetry. 
$\mathrm{C}_{8} \mathrm{~K}$ has graphene sheets, and each sheet is likely to become superconducting below the critical temperatures $T_{\mathrm{c}}$. The numbers of "electrons" and "holes" depend on the environments arising from the lattice structures. Since the lattice structures are very different in $\mathrm{C}_{8} \mathrm{~K}$ and $\mathrm{C}_{6} \mathrm{Ca}$, the critical temperatures should be different significantly.

BCS [5] used the fermionic pair operator equation.

$$
B_{k 0}^{2} \equiv B_{k 0} B_{k 0}=0
$$

constructed a ground-state vector and obtained a ground-state energy of an electron-phonon system:

$$
W_{0}=2 N w_{0},
$$

where $N$ is the pairon number per spin and $w_{0}$ the ground-state energy of the pairon, see Equation (17). The center-of-mass (CM) of the pairons move as bosons. That is, the eigenvalues of the pairon number operator $n_{q}$ are unlimited:

$$
n_{q}^{\prime}=0,1,2, \cdots,
$$

which is shown below.

The number operator in the $\boldsymbol{k}-\boldsymbol{q}$ representation

$$
n_{k q} \equiv B_{k q}^{\dagger} B_{k q}
$$

has eigenvalues 0 or 1: [13]

$$
n_{k q}^{\prime}=0 \text { or } 1 .
$$

The total number of a system of pairons, $N$, is represented by

$$
N \equiv \sum_{k} \sum_{q} n_{k q}=\sum_{q} n_{q},
$$

where

$$
n_{q} \equiv \sum_{k} n_{k q}-\sum_{k} B_{k q}^{\dagger} B_{k q}
$$

represents the number of pairons having net momentum $\boldsymbol{q}$. From Equations (30)-(32) we can establish Equation (28). To explicitly see this property, we introduce

$$
B_{q} \equiv \sum_{k} B_{k q}
$$

and obtain, after simple calculations,

$$
\left[B_{q}, n_{q}\right]=\sum_{k}\left(1-n_{k+\frac{1}{2} q}-n_{-k+\frac{1}{2} q}\right) B_{k q}=B_{q}, \quad\left[n_{q}, B_{q}^{\dagger}\right]=B_{q}^{\dagger} .
$$

Although the occupation number $n_{q}$ is not connected with $B_{q}$ as $N_{q} \neq B_{q}^{\dagger} B_{q}$, the eigenvalues $n_{q}^{\prime}$ of $n_{q}$ satisfying Equation (34) can be shown straightforwardly to yield Equation (28) with the eigenstates $|0\rangle$, $|1\rangle=B_{q}^{\dagger}|0\rangle,|2\rangle=B_{q}^{\dagger}|1\rangle=B_{q}^{\dagger} B_{q}^{\dagger}|0\rangle, \cdots$.

The present author's group [7] [8] [13] regards the superconductivity as a result of the BEC of the c-bosons. The free c-bosons moving in 2D with the linear dispersion relation $\varepsilon=c p$ undergoes a BEC at [13]

$$
k_{\mathrm{B}} T_{\mathrm{c}}=1.954 \hbar c n_{0}^{1 / 2},
$$

where $n_{0}$ is the pairon number density. The derivation of the linear dispersion relation $\varepsilon=c p$ and the BEC is outlined in Appendix. The average interpairon distance

$$
r_{0} \equiv n_{0}^{-1 / 2}
$$

is greater several times than the BCS coherence length (pairon size):

$$
\zeta_{0} \equiv h v_{\mathrm{F}} / \pi \Delta_{0},
$$

where $\Delta_{0}$ is the zero temperature BCS energy gap. We have 


$$
r_{0}=6.89 \zeta_{0} .
$$

Thus 2D pairons do not overlap in space. Hence the $T_{\mathrm{c}}$ can be calculated based on the free moving pairons model. See Ref. 12 for more details.

Formula (25) is distinct from the BCS formula in the weak coupling limit:

$$
2 \Delta_{0}=3.53 k_{\mathrm{B}} T_{\mathrm{c}} \text {. }
$$

Our Formula (35) obtained after identifying superconducting temperature as the BEC condensation temperature contains familiar quantities, the Fermi speed $v_{\mathrm{F}}$ and the boson density $n_{0}$ only.

For illustration let us take GaAs/AlGaAs. We assume $m^{*}=0.067 m_{\mathrm{e}}$ and $v_{\mathrm{F}}=1.36 \times 10^{8} \mathrm{~m} \cdot \mathrm{s}^{-1}$, then we obtain $n_{0}=10^{11} \mathrm{~cm}^{-2}, T_{\mathrm{c}}=1.29 \mathrm{~K}$ (reasonable). Not all electrons are bound with fluxons since the simultaneous generation of $\pm \mathrm{c}$-bosons is required. The plateau width vanishes at $T_{\mathrm{c}}$ since the energy gap vanishes there.

The neutral supercondensate is generated from the two ranges of energies of "electrons" and "holes". Hence it is difficult to precisely determine the critical temperature from the theoretical consideration alone. The comparison between theory and experiment may be carried out as follows. First we find the Fermi speed $v_{\mathrm{F}}^{(j)}$ from the Hall effect measurements or others. We then find the supercondensate density $n_{0}$ from the measurered critical temperature $T_{\mathrm{c}}$ by using Equation (35). In the mean field approximation we obtain

$$
-w_{0}=2 \varepsilon_{\mathrm{g}}(0)=k_{\mathrm{B}} T_{\mathrm{c}},
$$

which indicates a close connection between the zero temperature gap $\varepsilon_{\mathrm{g}}(0)$ and the critical temperature $T_{\mathrm{c}}$. A rigorous treatment of the BEC of free pairons shows a phase transition of the third order [13]. The molar heat rises like $T^{2}$, reaches $4.38 R\left(R=\right.$ gas constant) at $T_{\mathrm{c}}$, and then decreases to $2 R$ in the high-temperature limit [14] as shown in Figure 4. We note that the molar heat does not vanish above $T_{\mathrm{c}}$.

\section{Summary and Discussion}

We have developed a theory regarding $\mathrm{MgB}_{2}$ as a member of GIC. We start with the lattice configuration with all ions locations specified, and find that each B-plane contains B's forming a honeycomb lattice of the nearest neighbour distance (lattice constant) $a_{0}$ while each Mg-plane contains a base-hexagonal lattice of the lattice constant $\sqrt{3} a_{0}$. Since the B-lattice is more compact, it becomes superconducting at the lowest temperatures.

We obtain a linear dispersion relation $\varepsilon=c p$ for the moving pairons. The superconducting temperature $T_{\mathrm{c}}$, identified as the BEC temperature of the pairons, is given by $k_{\mathrm{B}} T_{\mathrm{c}}=1.954 \hbar c n_{0}^{1 / 2}$. The supercurrent density is

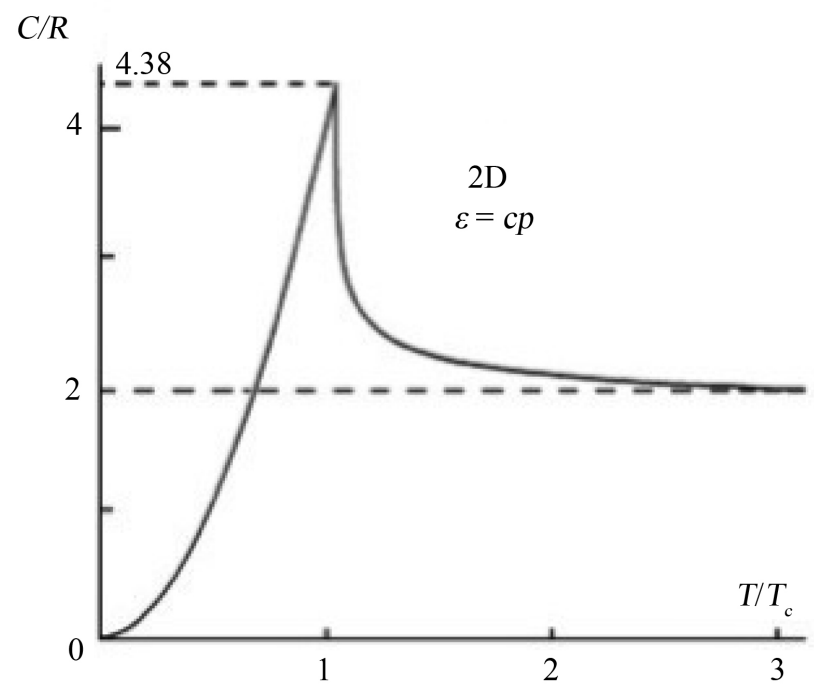

Figure 4. The molar heat capacity $C$ for $2 \mathrm{D}$ free massless bosons (after Ref. 14, Figure 6.3). 
calculated without introducing the averaging. The superconducting energy gap $\varepsilon_{\mathrm{g}}$ is identified as the gap in the pairon energy spectrum, distinct from the BCS energy gap $\Delta$.

Canfield and Crabtree have discussed two energy gaps, which is strange since there is one superconducting state at $0 \mathrm{~K}$. We shall discuss this topic in a separate publication.

\section{References}

[1] Nagamatsu, J., Nakagawa, N., Muranaka, T., Zenitani, Y. and Akimitsu, J. (2001) Nature, 410, 63. http://dx.doi.org/10.1038/35065039

[2] Canfield, P.C. and Crabtree, G.W. (2003) Physics Today, 56, 34. http://dx.doi.org/10.1063/1.1570770

[3] Hinks, D.G., Claus, H. and Jorgenson, J.D. (2001) Nature, 411, 457. http://dx.doi.org/10.1038/35078037

Kortus, J., et al. (2001) Physical Review Letters, 86, 4656. http://dx.doi.org/10.1103/PhysRevLett.86.4656

[4] Uchida, S. (2015) High Temperature Superconductivity. Springer, Japan, 9.

[5] Bardeen, J., Cooper, L.N. and Schrieffer, J.R. (1957) Physical Review, 108, 1175. http://dx.doi.org/10.1103/PhysRev.108.1175

[6] Ashcroft, N.W. and Mermin, N.D. (1976) Solid State Physics. Saunders, Philadelphia, 6-7, 216-217, 228-229.

[7] Fujita, S., Takato, Y. and Suzuki, A. (2011) Modern Physics Letters B, 25, 223. http://dx.doi.org/10.1142/S021794911025675

[8] Fujita, S. and Suzuki, A. (2010) Journal of Applied Physics, 107, 013711. http://dx.doi.org/10.1063/1.3280035

[9] Kang, N., Lu, L., Kong, W.J., Hu, J.S., Yi, W., Wang, Y.P., Zhang, D.L., Pan, Z.W. and Xie, S.S. (2003) Physical Review B, 67, 033404. http://dx.doi.org/10.1103/PhysRevB.67.033404

[10] Zhang, S.C., Hansson, T.H. and Kivelson, S. (1989) Physical Review Letters, 62, 82. http://dx.doi.org/10.1103/PhysRevLett.62.82

[11] Ehrenfest, P. and Oppenheimer, J.R. (1931) Physical Review, 37, 333. http://dx.doi.org/10.1103/PhysRev.37.333 Bethe, H.A. and Jackiw, R.W. (1989) Intermediate Quantum Mechanics. 2nd Edition, Benjamin, New York, 23. Fujita, S. and Morabito, D.L. (1998) Modern Physics Letters B, 12, 1061.

[12] Cooper, L.N. (1956) Physical Review, 104, 1189. http://dx.doi.org/10.1103/PhysRev.104.1189

[13] Fujita, S. and Godoy, S. (2001) Theory of High Temperature Superconductivity. Kluwer, Dordrecht, 54-58, 96-98, 107-109, 164-167, 230-231. http://dx.doi.org/10.1007/0-306-48216-9

[14] Fujita, S., Ito, K. and Godoy, S. (2009) Quantum Theory of Conducting Matter-Superconductivity. Springer, New York, 79-83. http://dx.doi.org/10.1007/978-0-387-88211-6 


\section{Appendix: Linear Dispersion Relation and Bose-Einstein Condensation}

We consider the case of a 2D superconductor. The phonon exchange attraction is in action for any pair of electrons near the Fermi surface. In general the bound pair has a net momentum, and hence, it moves. Such a pair is called a moving pairon. The energy $w_{q}$ of a moving pairon can be obtained from

$$
w_{q} a(\boldsymbol{k}, \boldsymbol{q})=\{\varepsilon(|\boldsymbol{k}+\boldsymbol{q} / 2|)+\varepsilon(|-\boldsymbol{k}+\boldsymbol{q} / 2|)\} a(\boldsymbol{k}, \boldsymbol{q})-\frac{v_{0}}{(2 \pi \hbar)^{2}} \int^{\prime} \mathrm{d}^{2} k^{\prime} a\left(\boldsymbol{k}^{\prime}, \boldsymbol{q}\right),
$$

which is Cooper's equation in 2D, Equation (1) of his 1956 Physical Review paper [12]. The prime on the $k^{\prime}$ -integral means the restriction on the integration domain arising from the phonon exchange attraction, see below. The pair wavefunctions $a(\boldsymbol{k}, \boldsymbol{q})$ are coupled with respect to the other variable $\boldsymbol{k}$, meaning that the exact (energy-eigenstate) pair wavefunctions are superpositions of $a(\boldsymbol{k}, \boldsymbol{q})$.

Equation (41) can be solved simply. We briefly review the calculations and results here. We assume that the energy $w_{q}$ is negative:

$$
w_{q}<0 \text {. }
$$

Then, $\varepsilon(|\boldsymbol{k}+\boldsymbol{q} / 2|)+\varepsilon(|-\boldsymbol{k}+\boldsymbol{q} / 2|)-w_{q}>0$. Rearranging the terms in Equation (41) and dividing by $\varepsilon(|\boldsymbol{k}+\boldsymbol{q} / 2|)+\varepsilon(|-\boldsymbol{k}+\boldsymbol{q} / 2|)-w_{q}$, we obtain from Equation (41)

$$
a(\boldsymbol{k}, \boldsymbol{q})=\frac{C(\boldsymbol{q})}{\varepsilon(|\boldsymbol{k}+\boldsymbol{q} / 2|)+\varepsilon(|-\boldsymbol{k}+\boldsymbol{q} / 2|)-w_{q}},
$$

where

$$
C(\boldsymbol{q}) \equiv \frac{v_{0}}{(2 \pi \hbar)^{2}} \int^{\prime} \mathrm{d}^{2} k^{\prime} a\left(\boldsymbol{k}^{\prime}, \boldsymbol{q}\right)
$$

is $k$-independent. Introducing Equation (43) in Equation (44), and dropping the common factor $C(\boldsymbol{q})$, we obtain

$$
1=\frac{v_{0}}{(2 \pi \hbar)^{2}} \int^{\prime} \frac{\mathrm{d}^{2} k}{\varepsilon(|\boldsymbol{k}+\boldsymbol{q} / 2|)+\varepsilon(|-\boldsymbol{k}+\boldsymbol{q} / 2|)+\left|w_{q}\right|} .
$$

We now assume a free-electron model in 2D. The Fermi surface is a circle of the radius (momentum)

$$
k_{\mathrm{F}} \equiv\left(2 m_{1} \varepsilon_{\mathrm{F}}\right)^{1 / 2}
$$

where $m_{1}$ represents the effective mass. The energy $\varepsilon(|\boldsymbol{k}|)$ is given by

$$
\varepsilon(|\boldsymbol{k}|) \equiv \varepsilon_{k}=\frac{|\boldsymbol{k}|^{2}-\left|\boldsymbol{k}_{\mathrm{F}}\right|^{2}}{2 m_{1}} .
$$

The prime on the $k$-integral in Equation (45) means the restriction:

$$
0<\varepsilon(|\boldsymbol{k}+\boldsymbol{q} / 2|), \varepsilon(|-\boldsymbol{k}+\boldsymbol{q} / 2|)<\hbar \omega_{\mathrm{D}} .
$$

We may choose the z-axis along $\boldsymbol{q}$ as shown in Figure 5 .

The $k$-integral in Equation (45) can then be expressed by

$$
\frac{(2 \pi \hbar)^{2}}{v_{0}}=4 \pi \int_{0}^{\pi / 2} \mathrm{~d} \theta \sin \theta \int_{k_{\mathrm{F}}+\frac{1}{2} q \cos \theta}^{k_{\mathrm{F}}+k_{\mathrm{D}}-\frac{1}{2} q \cos \theta} \frac{k^{2} \mathrm{~d} k}{\left|w_{q}\right|+2 \varepsilon_{k}+\left(4 m_{1}\right)^{-1} q^{2}},
$$

where $k_{\mathrm{D}}$ is

$$
k_{\mathrm{D}} \equiv m_{1} \hbar \omega_{\mathrm{D}} k_{\mathrm{F}}^{-1} .
$$

After performing the integration and taking the small- $q$ and small- $\left(k_{\mathrm{D}} / k_{\mathrm{F}}\right)$ limits, we obtain

$$
w_{q}=w_{0}+(2 / \pi) v_{\mathrm{F}} q
$$




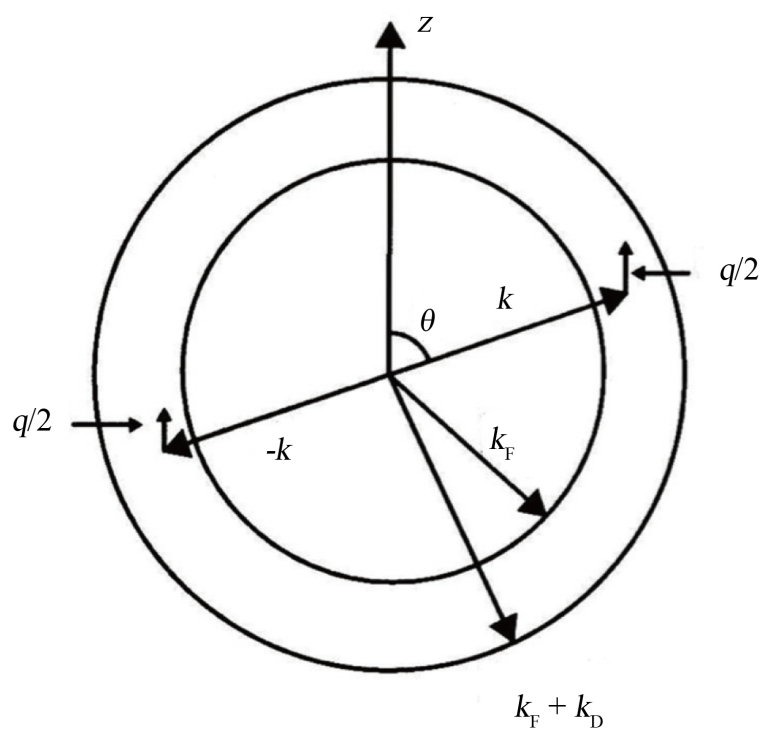

Figure 5. The range of the interaction variables $(k, \theta)$ is limited to a circular shell of thickness $k_{\mathrm{D}}$.

where

$$
w_{0}=\frac{-2 \hbar \omega_{\mathrm{D}}}{\exp \left\{2 / v_{0} \mathcal{D}(0)\right\}-1}
$$

is the pairon ground state energy.

As expected, the zero-momentum pair has the lowest energy $w_{0}$. The excitation energy is continuous with no energy gap. The energy $w_{q}$ increases linearly with momentum $q$ for small $q$. Hence, the Cooper pair moves like a massless particle with a common speed $(2 / \pi) v_{\mathrm{F}}$.

Such a linear dispersion relation is valid for pairs moving in any dimensions (D). However the coefficients slightly depend on the dimension as follows:

$$
w_{q}=w_{0}+c q
$$

where $c=(1 / 2) v_{\mathrm{F}}$ and $(2 / \pi) v_{\mathrm{F}}$ for 3 and $2 \mathrm{D}$, respectively.

The velocity $\boldsymbol{v}$ of the particle having a linear dispersion relation $\varepsilon=c p$ is defined and calculated as

$$
v_{x} \equiv \frac{\partial \varepsilon}{\partial p_{x}}=c \frac{\partial}{\partial p_{x}} p=c \frac{p_{x}}{p} .
$$

The velocity magnitude $|\boldsymbol{v}|$ is $c$. Hence, the pair moves with the speed $c$.

We consider a system of free bosons having a linear dispersion relation: $\varepsilon=c p=(2 / \pi) v_{\mathrm{F}}$ moving in $2 \mathrm{D}$. The system undergoes a Bose-Einstein condensation with the critical temperature $T_{\mathrm{c}}$ :

$$
k_{\mathrm{B}} T_{\mathrm{c}}=1.954 \hbar c n^{1 / 2}=1.244 \hbar v_{\mathrm{F}} n^{1 / 2},
$$

where $n$ is the $2 \mathrm{D}$ boson density. The $T_{\mathrm{c}}$ is proportional to the square root density. The derivation of Equation (55) is given in Ref. 13. Briefly, the chemical potential $\mu$ vanishes below $T_{\mathrm{c}}$ and decreases further above $T_{\mathrm{c}}$. The difference between the boson density $n$ and the zero momentum density $n_{0}$ is given by

$$
2 \pi \hbar^{2} c^{2} \beta^{2}\left(n-n_{0}\right)=\int_{0}^{\infty} \mathrm{d} x \frac{x}{\mathrm{e}^{-\beta \mu} \mathrm{e}^{x}-1},
$$

where $x \equiv \beta \varepsilon, \beta \equiv\left(k_{\mathrm{B}} T\right)^{-1}$. We put $\mu=0$ on the right-hand side and $\beta_{\mathrm{c}}=\left(k_{\mathrm{B}} T_{\mathrm{c}}\right)^{-1}$ and $n_{0}=0$ on the left-hand side, and obtain Equation (55). 


\section{Submit or recommend next manuscript to SCIRP and we will provide best service for you:}

Accepting pre-submission inquiries through Email, Facebook, LinkedIn, Twitter, etc.

A wide selection of journals (inclusive of 9 subjects, more than 200 journals)

Providing 24-hour high-quality service

User-friendly online submission system

Fair and swift peer-review system

Efficient typesetting and proofreading procedure

Display of the result of downloads and visits, as well as the number of cited articles

Maximum dissemination of your research work

Submit your manuscript at: http://papersubmission.scirp.org/ 